\title{
Die Erforschung der Luxemburger Wortbildung als Aufgabe. Eine Projektvorstellung
}

\author{
Heinz Sieburg und Britta Weimann (Luxemburg)
}

\begin{abstract}
Research on Luxembourgish word formation - both from a historical and a synchronic perspective - is still at its very beginning. Its most challenging aspect is the complex multilingual situation in Luxembourg which arose due to the country's intricate history. The research project "WBLUX" takes this unique situation into account; It has been designed as a multi-stage corpus-based tandem project as a cooperation between German and Luxembourgish studies. This paper commences by introducing the project design and the corpus concept, followed by a presentation of some exemplary corpus data.
\end{abstract}

\section{$1 \quad$ Einleitung}

Das Luxemburgische entwickelt sich seit dem 19. Jh. zunehmend zu einer eigenständigen Nationalsprache. Grundlage hierfür ist vor allem das Moselfränkische (Mslfrk.), das als zunächst nur gesprochene Varietät neben der (auch) schriftsprachlichen deutschen Sprache stand. Vorläufer für beide ist in weiten Teilen eine deutsch-moselfränkisch-luxemburgische Regionalsprache, die sich in der Schriftlichkeit bis ins Mittelalter zurückverfolgen lässt. ${ }^{1}$ Aus sprachhistorischer Sicht erscheint der moselfränkisch-luxemburgische Raum schon aufgrund dieser spezifischen Dynamik von besonderem Interesse. Dem trägt das an der Universität Luxemburg angesiedelte Forschungsprojekt „Die Wortbildung des moselfränkisch-luxemburgischen Raumes (WBLUX)“ Rechnung. Es ist als interdisziplinäres Tandemprojekt der Germanistik und Luxemburgistik konzipiert und in zwei Bearbeitungsphasen gegliedert:

1. Phase: Historische Wortbildung des moselfränkisch-luxemburgischen Raumes (WBLUX1, 2009-2012)

2. Phase: Die Wortbildung des Luxemburgischen. Historische Voraussetzungen und kontrastive Analyse (WBLUX2, 2013-2017)

Das Projekt zielt darauf ab, die (deutsch-luxemburgische) Schriftsprachlichkeit seit dem Mittelalter zu erschließen. Hierzu dient die Bildung eines Korpus, das in Hinblick auf die Wortbildung, konzentriert auf den Wortbildungstyp der expliziten Derivation, ausgewertet wird. Die Auswahl der Wortbildung als Gegenstand des Projektes rührt einerseits aus der Tatsache, dass

\footnotetext{
${ }^{1}$ Zur Entwicklung des Deutschen in Luxemburg cf. Weimann (2013: 253-255).

Linguistik online 77, 3/16 - http://dx.doi.org/10.13092/lo.77.2907

CC by 3.0
} 
die Wortbildung des Luxemburgischen bisher nicht systematisch untersucht worden ist ${ }^{2}$, andererseits aus der besonderen Bedeutung der Wortbildung für den Sprachausbau, der durch die Erschließung neuer Domänen und Textsorten Ausbau und Modernisierung des Wortschatzes erfordert.

Zum besseren Verständnis der Projektrelevanz ist es sinnvoll, knapp die rezente Sprachsituation in Luxemburg zu skizzieren. Anschließend folgen eine Vorstellung des Korpus, der Projektkonzeption und -methode sowie die Präsentation einiger Textbeispiele.

\section{$2 \quad$ Sprachsituation in Luxemburg}

Luxemburg ist eines der Länder, für die Mehrsprachigkeit konstitutiv ist. Seit der Sprachgesetzgebung von 1984 ist eine Dreisprachigkeit formell festgeschrieben. Dabei firmiert das Luxemburgische als Nationalsprache, das Französische und Deutsche werden als Amtssprachen benannt, wobei dem Französischen exklusiv der Status der Gesetzessprache zugewiesen ist. De facto ist Luxemburgisch, obwohl der Ausbau als Schriftsprache voranschreitet, primär Medium der mündlichen Kommunikation unter autochthonen Luxemburgern, spielt aber auch als mündliche Integrationssprache und als Sprache der konzeptionell mündlichen neuen Medien (soziale Netzwerke, Chat, Foren, SMS, private E-Mails) eine immer größere Rolle (cf. Gilles 2011: 5058). Französisch ist eine frequente Sprache in der Alltagskommunikation, insbesondere auch im Kontakt mit den meisten ausländischen Einwohnern und Grenzpendlern des Großherzogtums und in vielen öffentlichen formellen Kommunikationssituationen. Das Deutsche ist dagegen primär Sprache der Printmedien (Zeitungen, Büchermarkt, Wissenschaftsliteratur) und des Fernsehens. Zunehmend relevant wird daneben das Englische; eine starke Position hat zudem das Portugiesische, zumindest innerhalb der bedeutsamen Gruppe portugiesischer Immigranten.

Der „Sprachenmarkt“" Luxemburgs ist eng verkoppelt mit der Bevölkerungszusammensetzung des Großherzogtums und vor allem mit dem hochdynamischen Arbeitsmarkt. Die Gesamteinwohnerzahl Luxemburgs beläuft sich auf ca. 550'000 (cf. STATEC 2014: 9), eingerechnet der vergleichsweise hohe Ausländeranteil von ca. 45\% (cf. STATEC 2014: 9). Typisch für Luxemburg ist zudem eine große und stetig steigende Zahl von Grenzpendlern. Derzeit beläuft sich diese auf rund 160'000, davon rund die Hälfte aus dem angrenzenden Frankreich, jeweils etwa ein Viertel aus dem ebenfalls benachbarten Belgien und Deutschland kommend (cf. STATEC 2014: 12).

Deutsch und Französisch sind seit dem Mittelalter die beiden prägenden Sprachen Luxemburgs, ihr heutiger Status ist allerdings durchaus problematisch, zumindest insofern, als sie von der Mehrheit der Luxemburger nicht als Muttersprachen angesehen werden, andererseits aber auch nicht eigentlich als Fremdsprachen aufgefasst werden können. Historisch betrachtet unterlag das Mehrsprachigkeitsgefüge Luxemburgs unterschiedlichsten Außeneinflüssen und fremdgesteuerten Sprachlenkungsmaßnahmen, die oft einhergingen mit der Vereinnahmung des Landes

\footnotetext{
${ }^{2}$ Neben Arbeiten zu einzelnen Affixen und Funktionen bieten Russ (2000) und Sánchez Prieto (2008) einen ersten Überblick über die Wortbildung des Luxemburgischen. Hinweise zur Wortbildung finden sich auch in der dreibändigen Grammatik von Schanen/Zimmer (2005/2006).
} 
von französischer, niederländischer, vor allem aber deutscher Seite. Diese historischen Erfahrungen wirken auf das Sprachwertsystem bis heute nach.

Sprachhistorisch und dialektgeografisch gehört Luxemburg, das im Zuge des Londoner Vertrages 1839 seine romanophonen Landesteile an Belgien abgetreten hat, dem deutschen Sprachraum an - so jedenfalls die traditionelle Auffassung - und ist hier als Teil des Westmitteldeutschen bzw. des Moselfränkischen aufzufassen, cf. Abbildung 1.

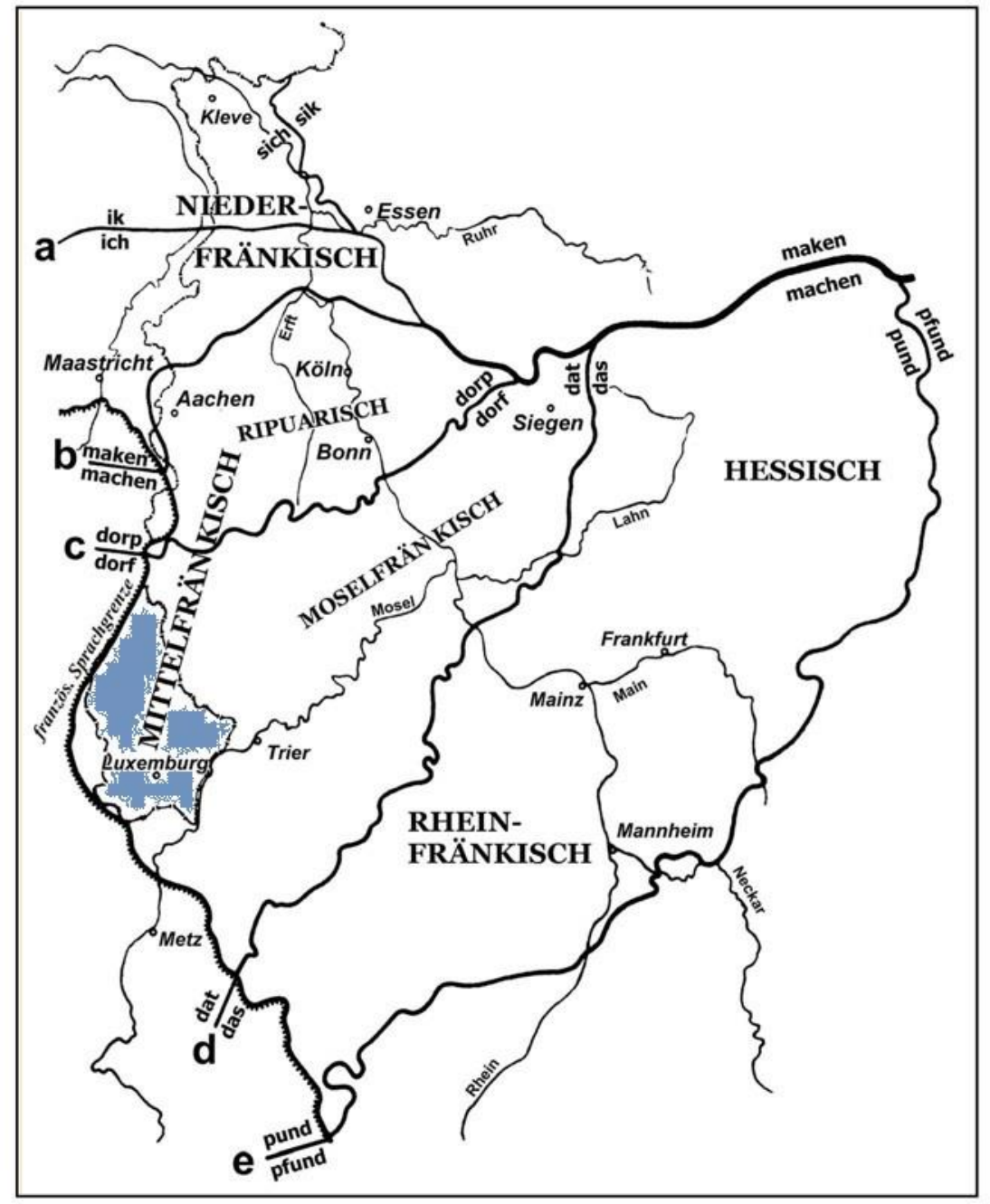

Abbildung 1: Westmitteldeutsch (Paul, Mhd. Gr. § E 5)

Unter dieser Voraussetzung ist es durchaus konsequent, dass Luxemburg etwa im Zuge der Erhebungen zum deutschen Sprachatlas miterfasst wurde oder auch im Band zur mittelhochdeutschen Wortbildung von Klein/Solms/Wegera (2009) berücksichtigt ist.

Allerdings lässt sich die Frage stellen, ob ein Perspektivenwechsel und eine Fokusverschiebung hin zu einer dezidiert Luxemburger Sicht nicht beschreibungsadäquater wären. Fest steht jedenfalls, dass die Klassifizierung des Luxemburgischen als Dialekt des Deutschen - auch wenn das bisweilen immer noch vorfindbar ist - an den linguistischen Realitäten vorbeigeht. Kriterien wie Normenkodifikation, (zunehmende) Schriftsprachlichkeit, aber auch der Status als Nationalsprache sind mit den für Dialekte gültigen Definitionskriterien nicht vereinbar, lassen sich

\footnotetext{
${ }^{3}$ Farbliche Hervorhebung nicht im Original.
} 
aber sehr wohl mit dem von Heinz Kloss (1978) entwickelten Konzept einer Ausbausprache verbinden. Das Luxemburgische ist heute kein Dialekt des Deutschen mehr, sondern eine eigenständige Nationalsprache neben dem Deutschen. So wird es auch von einer deutlichen Mehrheit (83\%) der Einwohner Luxemburgs gesehen, wie eine repräsentative Umfrage im Rahmen des interdisziplinären Projekts „IDENT2“ an der Universität Luxemburg ergeben hat (cf. Sieburg/Weimann 2014: 358-361).

Eine solche Bestimmung bietet zugleich einen legitimen Ansatzpunkt dafür, auch die sprachhistorische Forschung Luxemburgs ,,auf eigene Füße zu stellen“, zumal nur so die spezifische Situation des Sprachraumes und seine besondere Dynamik angemessen beschrieben werden können. Dieser Sichtweise ist auch das hier vorzustellende Wortbildungsprojekt verpflichtet, das idealiter ein Schritt auf dem Weg zu einer umfassenderen Sprach(en)geschichte Luxemburgs sein soll. Die Erforschung der Wortbildung ist insofern als ein Mosaikstein eines größeren Ganzen zu sehen.

Aber bereits hier ergeben sich eine Reihe von Implikationen und Fragestellungen. Als Herausforderung stellt sich etwa die angemessene terminologische Bezeichnung der zu untersuchenden historischen Varietät dar. Die Bezeichnung „Deutsch“ („Althochdeutsch“, „Mittelhochdeutsch“, „Frühneuhochdeutsch“) ist aus sprachtypologischen Gründen einerseits nachvollziehbar, verdeckt aber das Arealtypische der zu untersuchenden Varietät. Auch „Moselfränkisch" greift im Grunde noch zu weit, weil der Luxemburger Sprachraum nur einen Ausschnitt daraus bildet. Von daher würde sich die Bezeichnung „Luxemburgisch“ bzw. - zur Kenntlichmachung der historischen Vorstufe - „Altluxemburgisch“ anbieten, wobei freilich die enge Verflechtung mit den östlich angrenzenden deutschen Varietäten durch diesen Begriff nicht geleugnet und verdeckt werden soll.

\section{$3 \quad$ Korpus und Untersuchungsgebiet}

Die komplexe Entwicklungsgeschichte der beiden heute im Untersuchungsgebiet verwendeten Sprachen Deutsch und Luxemburgisch und ihrer gemeinsamen historischen Vorstufe ist nicht die einzige Schwierigkeit bei der Erstellung des Korpus; auch der lange Untersuchungszeitraum von etwa 800 Jahren, der sich vom Mittelalter bis zur Gegenwart erstreckt und bedeutende Umbrüche der politischen, sozialen und medialen Strukturen einschließt, spiegelt sich in den überlieferten und damit für das Korpus zur Verfügung stehenden Texten wider. Der besonderen Situation der Ausgliederung von Varietäten (die luxemburgischen Dialekte) aus dem Varietätengefüge einer Sprache (Deutsch, cf. Gilles 1998) und der Entstehung und Etablierung einer neuen Standardvarietät (Luxemburgisch) trägt die Projektkonzeption mit ihrem Zwei-PhasenModell Rechnung, indem für die Zeit der regionalsprachlich-moselfränkischen und später deutschen Schriftsprachlichkeit - als eine Art „Vorgeschichte“4 der luxemburgischen Sprache - und für die Zeit der Entstehung des Luxemburgischen je eigene (Teil-)Korpora gebildet werden.

Während das Untersuchungsgebiet für Korpus 2 (19. bis 21. Jh.) sich auf Luxemburg in seinen heutigen, seit 1839 bestehenden Grenzen erstreckt, wird für Korpus 1, das die Zeit von 1200 bis zum Ende des 18. Jahrhunderts abdeckt, ein größerer Raum angenommen, der ungefähr der

\footnotetext{
${ }^{4}$ Vorgeschichte meint hier die Zeit, bevor die moselfränkisch-luxemburgische Schriftlichkeit zu Beginn des 19. Jahrhunderts wieder einsetzt und sich schließlich zu einer eigenen Standardvarietät entwickelt.
} 
historischen Grafschaft bzw. dem Herzogtum Luxemburg (ab 1354) entspricht. Abbildung 2 zeigt die Abweichungen zu den heutigen Grenzen. Die orange gefärbten Flächen im Südwesten und Süden gingen 1659 an Frankreich ${ }^{5}$, die gelb eingefärbten im Osten 1815 an Preußen ${ }^{6}$ und die grünen im Westen 1839 an Belgien. In allen drei Fällen waren zumindest auch germanophone Gebiete betroffen. Die romanisch-germanische Sprachgrenze verlief weitgehend entlang der heutigen Westgrenze Luxemburgs (rot gestrichelt), im Süden aber westlich der seit dem Londoner Vertrag von 1839 zu Belgien gehörenden Gegend um Arlon.

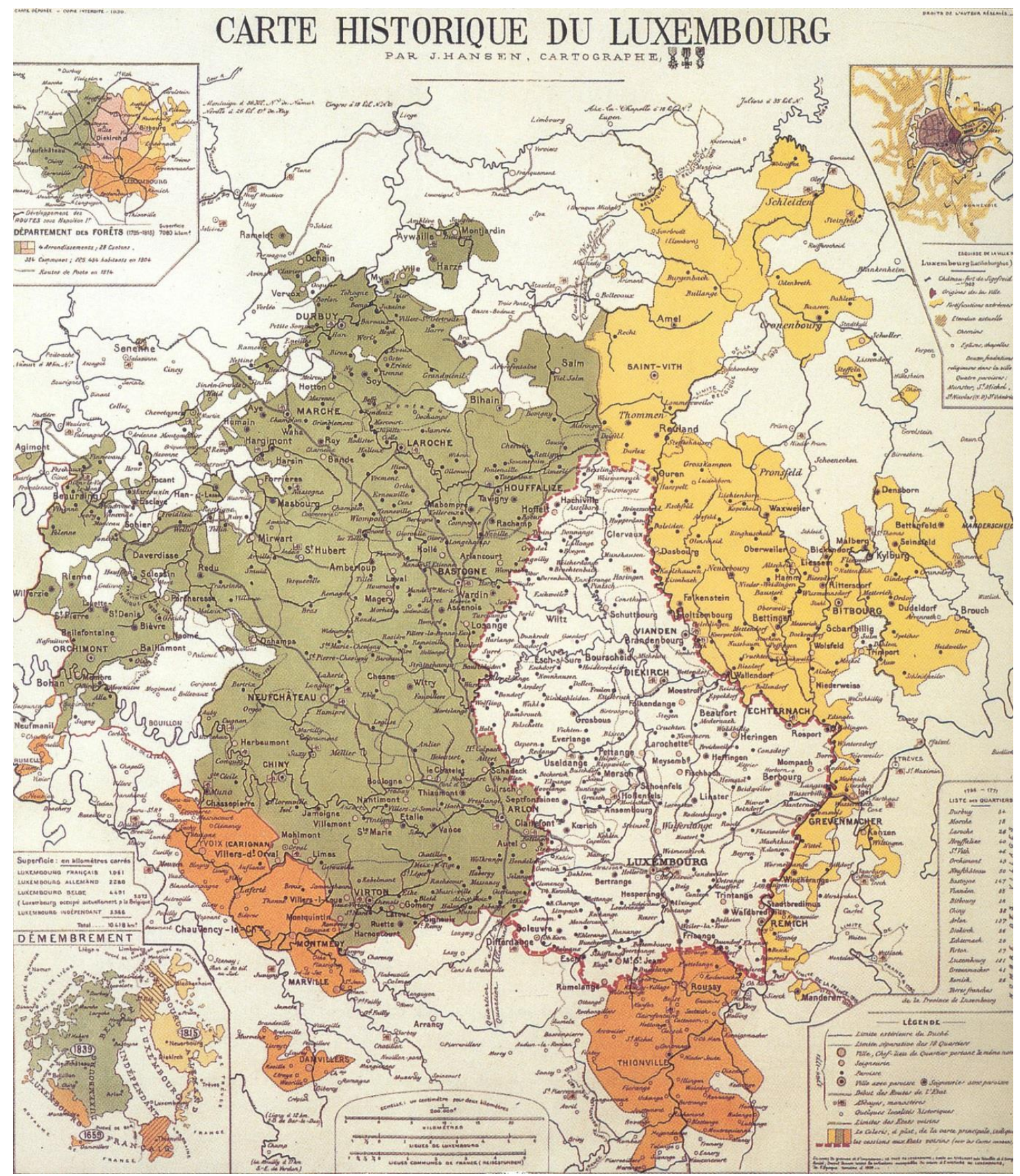

Abbildung 2: Historische Karte Luxemburgs (Joseph Hansen 1930)

\footnotetext{
${ }^{5}$ Zur Umsetzung der Gebietsveränderungen von 1659 in der vorliegenden Karte cf. Uhrmacher (2010) mit einem Alternativvorschlag in Form einer Kartenserie.

${ }^{6}$ Die Gebietsabtretungen sind in der kleinen Karte unten links genauer aufgeschlüsselt. Die gelb schraffierten Gebiete gehören heute zu Belgien, die unschraffierten gelben zu Deutschland.
} 
Auch mit den später an die Nachbarstaaten abgetretenen Gebieten ist der Untersuchungsraum relativ begrenzt, die Überlieferungslage besonders im Mittelalter schwierig. ${ }^{7}$ Dazu kommt v. a. bei den Handschriften das Problem verfügbarer Editionen. Der erste zusammenhängende Text und damit älteste Korpustext ist der „Gothaer Fiebersegen“, der um 1200 (cf. Palmer 1991: 216) in Echternach geschrieben wurde. ${ }^{8}$ Noch älter sind nur die ebenfalls in der Abtei Echternach eingetragenen Glossen des 8. bis 11. Jahrhunderts, die u. a. wegen ihrer Überlieferungssituation und teilweise strittigen schreibsprachlichen Zuordnung ausgeschlossen wurden. Da für das 13. Jh. keine weiteren Texte aus dem Untersuchungsgebiet zur Verfügung stehen, wurden vier kürzere moselfränkische Texte (,Albanus“, „Christi Geburt“, „Herzog Ernst A“, „Rolandslied-Fragment") und moselfränkische Urkunden zum Vergleich hinzugezogen. Auch in den späteren Jahrhunderten sind im Untersuchungsgebiet nur wenige längere Texte überliefert wie Bruder Hermanns „Leben der Gräfin Yolanda von Vianden“, um 1320/1330 im Kloster Marienthal geschrieben (cf. Gärtner 2001: 46), so dass Textserien aus den Bereichen Recht und Verwaltung die Lücken füllen. Im 14. Jh. beginnen die umfangreichen Serien Echternacher Urkunden (ab 1348) und der Rechnungsbücher der Stadt Luxemburg (ab 1388), im 15. Jh. sind erste Weistümer und ähnliche ländliche Rechtstexte überliefert. Die ersten Drucke aus Luxemburg stammen wie die handschriftlichen Hexenprozessakten aus dem späten 16. Jh. Handschriftliche Weistümer sind parallel noch im 17. Jh. überliefert. Für das 18. Jh. bietet das Korpus bis auf die handschriftlichen Aufzeichnungen des Echternacher Mönchs Placidus Eringer von 1682-1728 (cf. Schiltz/Kauthen 2009) ausschließlich Drucke. Wie schon im 17. und späten 16. Jh. sind das neben Verordnungen und anderen Texten der Verwaltung v. a. religiöse Texte (Schriften der Jesuiten, Katechismus). Bei den Drucken wurden mit Matthias Birthon, Hubert Reulandt und Johann Baptist Kleber drei Drucker der Stadt Luxemburg, bei den Hexenprozessakten mit Hamm, Neuerburg (beide heute Rheinland-Pfalz), Echternach und Koerich vier Gerichtsorte, sowie bei den Weistümern mit der Stadt Luxemburg, Echternach, Frisingen, Asselborn, Koerich, Ettelbrück und Junglinster sieben Orte ${ }^{9}$ ausgewählt. Tabelle 1 zeigt die zeitliche Verteilung der Textserien aus Recht und Verwaltung:

\footnotetext{
${ }^{7}$ Zur Überlieferung des Moselfränkischen von den Anfängen bis zum Ende des 14. Jahrhunderts cf. Weimann (2012: 10-14).

${ }^{8}$ Zur Überlieferung und $\mathrm{zu}$ weiterer Literatur cf. Handschriftencensus (http://www.mr1314.de/1995 [24.02.2015]).

${ }^{9}$ Bei der Auswahl der Orte spielte neben der vorhandenen Überlieferung auch die Zugehörigkeit zu den vier Dialektgebieten Luxemburgs (cf. die Karte in Gilles 1999: 62) eine Rolle.
} 


\begin{tabular}{|c|c|c|c|c|c|c|}
\hline \multirow{3}{*}{$\begin{array}{l} \\
\text { Urkunden }^{10} \\
\text { Rechnunoshïch }\end{array}$} & 13. Jh. & 14. Jh. & 15. Jh. & 16. Jh. & 17. Jh. & 18. Jh. \\
\hline & & & & & & \\
\hline & & & & & & \\
\hline Weistümer $^{11}$ & & & & & & \\
\hline Hexenprozessa & & & & & & \\
\hline Verordnungen & & & & & & \\
\hline
\end{tabular}

Tabelle 1: Texte aus Recht und Verwaltung in Korpus 1

Hinsichtlich der schreibsprachlichen Entwicklung ist Korpus 1 zweigeteilt: Eine überwiegend moselfränkische Schreibsprache mit zunächst nur einzelnen, später zunehmenden südlichhochdeutsch geprägten Einflüssen, wie sie für den moselfränkischen Sprachraum typisch sind, zeigen neben den Urkunden und Rechnungsbüchern auch die wenigen Einzeltexte des 13. bis 15. Jahrhunderts, wie die bereits erwähnte Yolanda-Dichtung Bruder Hermanns:

So woilde ich bỳt der helfen din

Celÿhte brÿngen mohte ỳt fin

Ein wolkelofen morge roid

Den nog dy் werld nog der doid

Nog nid nog haz gebergen mach

Dý wile ich leuen einen dag

Bruder Hermann (von Veldenz): Leben der Gräfin Yolanda von Vianden, Vers 7-12

(Moulin 2009: 19)

Schon mit Blick auf $\dot{y} t$ (mhd. $\ddot{z} z$ ) mit unverschobenem wgerm. ${ }^{*} t$ und helfen mit verschobenem wgerm. * $p$ nach *l lässt sich der Text als moselfränkisch erkennen. Karte 1 zeigt die zu vergleichenden dorp-dorf- und dat-das-Isoglossen, die das Mslfrk. nach Norden hin zum Ripuarischen und nach Süden hin zum Rheinfränkischen und Hessischen abgrenzen. Weitere allgemein mittelfränkische (mfrk.) Kennformen (cf. Klein 2000: 15-20) sind bit für mit, die Digraphie <oi> u. a. für mhd. $\hat{o}$ (roid, mhd. rôt; doid, mhd. tôt), $<\mathrm{g}>$ für den Frikativ in nog (mhd. noch), unverschobenes wgerm. *d (doid; dag, mhd. tac) und die Frikativschreibung $<\mathrm{u}>$ in leuen für den Fortsetzer von wgerm. $* \hbar$ (mhd. lëben).

Dagegen vertreten die Weistümer, Hexenprozessakten und generell alle Drucke des Korpus eine weitgehend hochdeutsche Schreibsprache mit wenigen moselfränkischen Resten. Die Texte dieser zweiten Gruppe stammen bis auf einige Weistümer des späten 15. Jahrhunderts aus dem 16. bis 18. Jh. Die schreibsprachliche Zweiteilung von Korpus 1 korreliert also mit Zeit und Textsorte, den beiden wesentlichen Strukturkriterien des Gesamtkorpus. Der Vergleich des Luxemburger Rechnungsbuchs aus den Jahren 1499 bis 1500, das noch den regionalsprachlich-moselfränkischen Typ vertritt, mit einem Weistum aus Echternach, das von Mathias Hardt (1870: 174) anhand äußerer Kriterien auf den Zeitraum 1462-1539 datiert wird, verdeutlicht die schreibsprachlichen Unterschiede:

Dit ift Sulchs ich jacop buymeifter vurg(enannt)

vßgeben hain die doyn(n)en ain feillers port

\footnotetext{
${ }^{10}$ Die Urkunden des 13. Jahrhunderts (grauer Balkenabschnitt) stammen nicht aus dem engeren Luxemburger Umfeld und werden wie einige weitere moselfränkische Frühtexte nur zum Vergleich herangezogen.

${ }^{11}$ Die Weistümer des späten 15. und frühen 16. Jahrhunderts (grauer Balkenabschnitt) stehen in Zusammenhang mit dem Kloster St. Maximin in Trier.
} 
jn zu legen vnd zu mourre(n) vnd paffen dal bor(e)n zu befferen vnd ain das pofter trappen ain mourfeiltz als gefchr(iben) volget

Prymo jn dem Eue(n)maent So hait johan va(n) nurtzingen gearbet ain feillers port(en) die donnen jn zu müren ne(m)liche(n) viij dage vnd off paffendal bor(e)n zu befferen gearbet $\mathrm{v}$ dage [...]

Rechnungsbuch der Stadt Luxemburg 1499/1500 (LU 120 R 203), 14r

(Ravida 2012: Anhang S. 187)

Das $<\mathrm{b}>$ in $v \beta$ geben statt der regionalsprachlich zu erwartenden Frikativschreibung $<\mathrm{u}, \mathrm{v}>$ oder ift statt mfrk. is zeigt erste Ausgleichstendenzen hin zu einem südlich-hochdeutschen schreibsprachlichen Vorbild, wie sie für das 14. und 15. Jh. typisch sind. Daneben sind aber die moselfränkisch-regionalsprachlichen Charakteristika van für mhd. von, < dit> für mhd. diz, <ai> als Bezeichnung für einen Langvokal (hait, mhd. hât), unverschobene wgerm. *d (dage, mhd. tage) und (entlehntes) *p (paffen, mhd. phaffe) immer noch vorhanden. ${ }^{12}$

Diese artickell undt freyheitt sollen die scheffen zu Echternach bey ihren eyden in hueden halten von beyder hern undt burgerschafft.

1. Zum ersten, so einer ein richter oder ein scheffen wirdt, soll der scholtheis von wegen meines hern des abts, des fursten undt der burgerschafft inen vorhalten wie hernach geschrieben stehett.

Ihr richter oder scheffen gelobett meinem hern dem abt als einem rechten grundtlehnhern von wegen des lieben sant Wilbrorts gotteshauss getreuw undt holdt zu sein, das best zu werben undt das argst zu warnen, seine herligkeit als einem grondthern zu halten nach dem besten ihr kondt undt mucht.

Weistum von Echternach (LRA. A VII, 5.) (Majerus 1956: 123)

Im Vergleich mit dem Rechnungsbuch von 1499/1500 hat das Echternacher Weistum für wgerm. $* \hbar$ und $* d$ konstant die Ausgleichsformen $<\mathrm{b}>$ (geschrieben, gelobett) und $<\mathrm{t}>$ (gotteshauss), außerdem regelmäßige Diphthongierungsschreibungen ${ }^{13}$ (freyheitt, mhd. vrîheit; getreuw, mhd. getriuwe) und $<\mathrm{h}>$ (ihren, stehett) als Dehnungszeichen. Dem stehen nur noch wenige regionalsprachliche Charakteristika wie $<\mathrm{g}>$ als Frikativschreibung in herligkeit gegenüber.

Korpus 2 beginnt mit den Zeugnissen der in den 20er Jahren des 19. Jahrhunderts wiedereinsetzenden moselfränkischen Schriftlichkeit ${ }^{14}$ und führt bis zum rezenten Luxemburgischen.

\footnotetext{
${ }^{12}$ Für eine ausführliche Charakterisierung der Schreibsprache der Luxemburger Rechnungsbücher cf. Ravida (2012).

${ }^{13}$ In den Luxemburger Rechnungsbüchern wird die Diphthongierung der hohen Langvokale (mhd. $\hat{\imath}, i u, \hat{u}$ ) nur unregelmäßig bezeichnet. Das Alter und die Verbreitung dieses Lautwandels (Monogenese oder Polygenese) im Moselfränkischen sind umstritten. Cf. zur Diskussion Paul, Mhd. Gr. § L 17 und Weimann (2012: 17-20) mit einzelnen Frühbelegen aus der ersten Hälfte des 14. Jahrhunderts.

${ }^{14}$ Daneben gibt es in Luxemburg bis heute eine reiche deutsch- und französischsprachige Literatur.
} 
Das folgende Textbeispiel stammt aus dem ersten auf Luxemburgisch publizierten Buch $E$ ' Schrek ob de' Lezeburger Parnassus, einer 1829 erschienenen Lyriksammlung des Mathematikprofessors Antoine Meyer:

\section{OIEN D'KRISTIN}

KRISTIN och hei ob dem Klé

Dan dein Hïerzchen,

Balsem keemool mer sé

Ob mei' Schmïerzchen,

Oh Hïerzchen!

$[\ldots]$

Antoine Meyer: E' Schrek ob de' Lezeburger Parnassus

(Meyer 1829: 7)

Das luxemburgische Vorwort zur verwendeten Schreibweise ${ }^{15}$ und das ebenfalls luxemburgische Nachwort zur Grammatik können als erster luxemburgischer Sachtext gelten. Aus dem 19. Jh. liegen hauptsächlich Gedichte vor, aber auch zahlreiche Theaterstücke, v. a. von Edmond de la Fontaine unter dem Pseudonym Dicks veröffentlicht, und das Versepos Renert von Michel Rodange. Im 20. Jh. dominieren zunächst weiter Gedichte und Theaterstücke. Der erste luxemburgischsprachige Roman, D'Kerfegsblo'm von Adolf Berens, erscheint von 1921 bis 1928 in drei Bänden. Erst in den achtziger Jahren nimmt die Produktion luxemburgischsprachiger Romane spürbar zu. Das folgende Textbeispiel stammt aus dem 1990 erschienenen Roman Frascht von Nico Helminger:

tout fuit, tout se refuse à mes embrassements. wäre gudden ufank ${ }^{16}$. mais c'est du racine!

je sais, je c'est, je sais.

méng aarbecht geet jo doriwwer.

d'scheinwerfer vum bus sichen d'nuecht of, de mueren, dee sech mat enger blatzeger sträif ukënnegt, virun där schwaarz gerëpper vun eenzelne beem defiléieren, dann haiser, lut a verschiddene fënsteren, ziddreg nuetsfriichten vu bluttorange bis zitrounegiel. d'auer as stoe bliwwen, awer du weess, wéini de bus soll ukommen. de chauffeur huet d'saach am grëff, déi grouss rénkel, säi räichtum, säi räich. eng flaatsch niwwel, an du bas an de wollécken.

Nico Helminger: Frascht

(Helminger 1990: 7)

Korpus 2 enthält für jedes Jahrhundertviertel soweit möglich Texte aller drei Großgattungen, cf. Tabelle 2:

\footnotetext{
15 Eine verbindliche Orthografie existierte zu dieser Zeit noch nicht. Erst 1946 wird eine offizielle Orthografie eingeführt, die sich aber nicht durchsetzt und die daher 1975 umfassend sowie 1999 in geringerem Umfang reformiert wird (cf. Moulin 2006: 318-325).

${ }^{16}$ Im Luxemburgischen gilt eigentlich Substantivgroßschreibung.
} 


\begin{tabular}{ll|l|l|l} 
& & 19. Jh. & 20. Jh. & 21. Jh. \\
\hline Literatur & Epik & & & \\
& Lyrik & & \\
Drama & & & \\
Medien & Nachrichtenseite (rtl.lu) & & & \\
Politik & $\begin{array}{l}\text { Parlamentsprotokolle } \\
\text { politische Interviews }\end{array}$ & & & \\
Alltag & Interviews & &
\end{tabular}

Tabelle 2: Texte aus Korpus 2

Außerhalb der Literatur und der vereinzelten Fachtexte liegt mit den Parlamentsprotokollen eine interessante Textsorte vor, die Transkriptionen vorbereiteter Reden und spontansprachlicher Diskussionen vereint und über einen längeren Zeitraum verfügbar ist. Für das Korpus ausgewählt wurden drei Zeiträume: 1945-50, 1975-77 und 2002-2012. Für die Wortbildungsforschung sind die Parlamentsprotokolle v. a. wegen ihrer fachsprachlichen Debatten mit ihren besonderen Anforderungen an den Wortschatz einer noch jungen Sprache ergiebig. Ähnliche Herausforderungen stellen auch die Nachrichten der Internetseite rtl.lu für den Wortschatz des Luxemburgischen dar. Um Übersetzungen von Agenturmeldungen ausschließen zu können, wurden nur die nationalen Nachrichten ausgewertet.

Außerhalb von Korpus 2 steht eine umfangreiche Textsammlung zur Verfügung, die bei bestimmten Fragestellungen wie dem Einfluss des Deutschen auf die luxemburgische Wortbildung als Erweiterungskorpus dient. Sie enthält u. a. Leserkommentare der Seite rtl.lu, Protokolle von Jugendchats und Facebookgruppen, die Texte der luxemburgischen Wikipedia sowie Masterarbeiten, die im Studiengang „Master en langues, cultures et médias - Lëtzebuerger Studien“" der Universität Luxemburg entstanden sind.

\section{$4 \quad$ Programme und Annotationen}

Die Texte beider Korpora und des Erweiterungskorpus liegen elektronisch vor. Nach dem TEIStandard ${ }^{17}$ wurden die Texte von Korpus 1 und 2 bearbeitet. Annotationen zur Wortbildung wurden eigens für dieses Projekt entwickelt, allgemeinere Annotationen (Wortart, Genus etc.) lehnen sich an das für historische Sprachstufen des Deutschen entwickelte Tagset HiTS (cf. Dipper et al. 2013) an. Bei der Lemmatisierung wurden für Korpus 1 der Editor Anselmus ${ }^{18}$ von Stefan Müller, für Korpus 2 eigene Programme und Tools des Projektmitarbeiters Joshgun Sirajzade verwendet. Die unterschiedliche Verarbeitung der Texte aus den beiden Korpora ist durch ihren unterschiedlichen Normierungsgrad begründet. Die große Variation der Schreibungen in Korpus 1 wurde mit einem Lemmatisierungstool, das auf Ähnlichkeitsberechnungen basiert, bearbeitet, während in Korpus 2 eine teilautomatische Affixanalyse eingesetzt werden konnte, obwohl auch hier die orthografischen Unterschiede v. a. in den frühen Texten teils gravierend sind. Verbunden werden die Daten beider Korpora über eine Metalemmaliste. Abb. 3 und 4 zeigen Screenshots der beiden verwendeten Programme:

\footnotetext{
${ }^{17} \mathrm{Zu}$ den Zielen und Grundsätzen der Text Encoding Initiative cf. http://www.tei-c.org [24.02.2015].

18 Cf. Müller (2012) zu einer früheren Version des Editors.
} 
C:Lanselmus_ia $1499-1500$, z_lux, kon_lem_aut.html

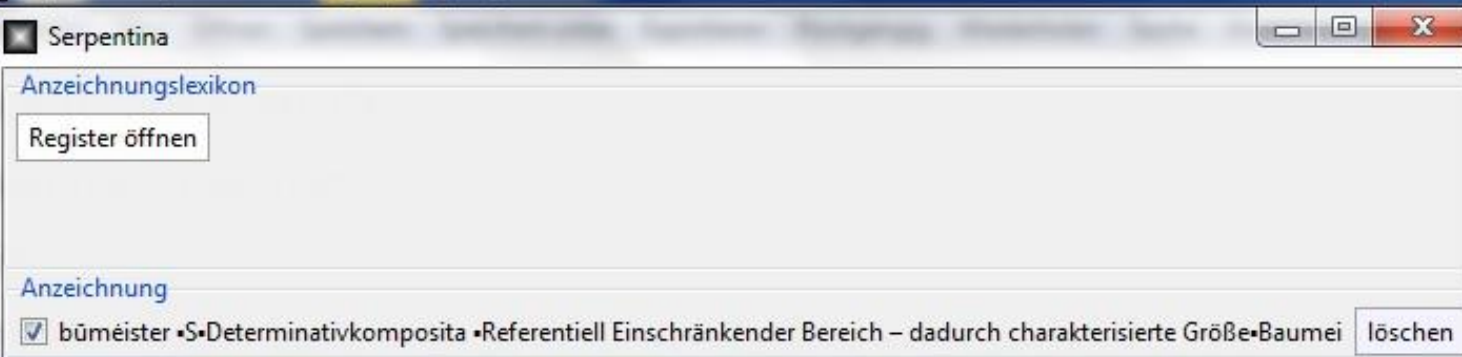

$\checkmark$ bümèister -S•Determinativkomposita $\cdot$ Referentiell Einschränkender Bereich - dadurch charakterisierte Größe•Baumei löschen

buymeirter vßgeben hain van foürloin den wech

$\langle$ dg $>$ den $</ d g>z u$ klu「en zu fullen vnd $\lceil$ tey $<k u ̈>n</ k u ̈>\lceil a u e l$ vnd

Abbildung 3: der Editor Anselmus

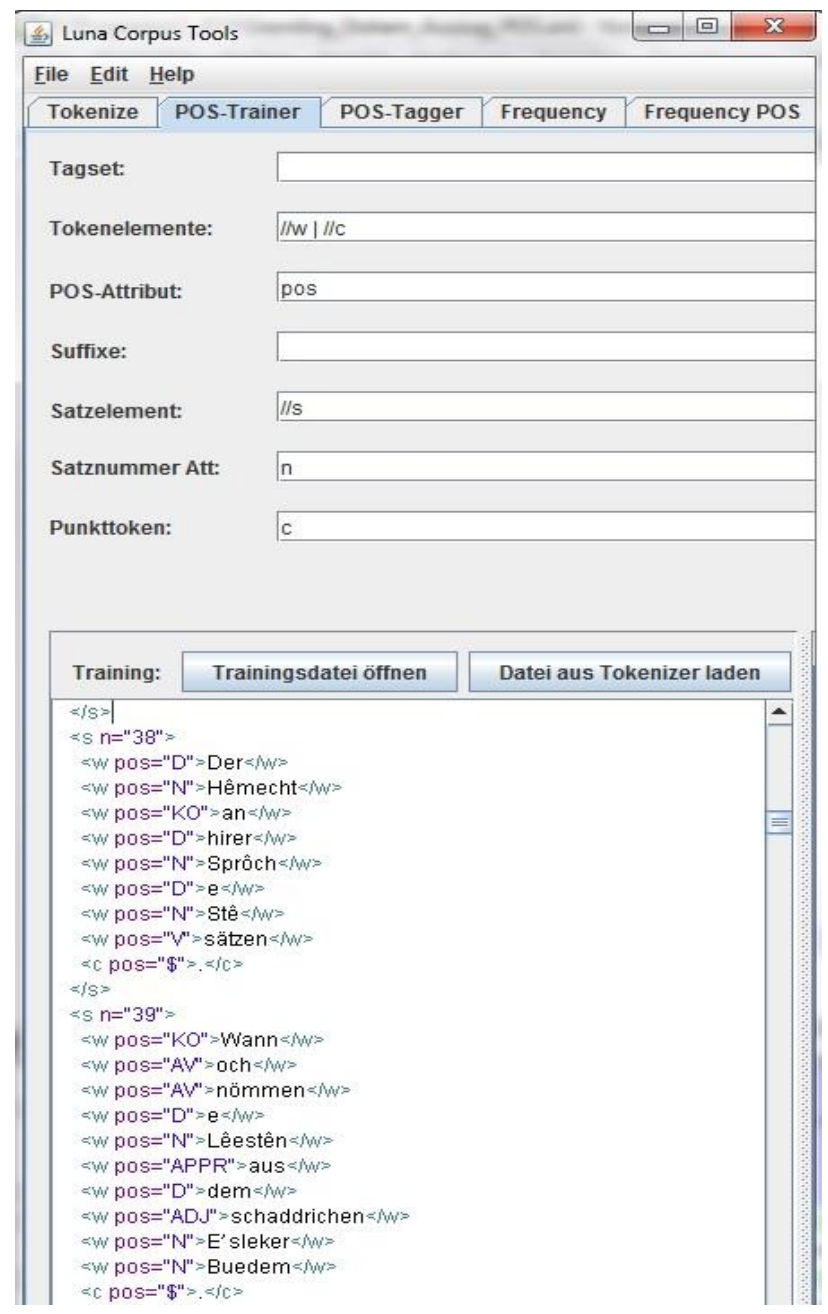

Abbildung 4: Luna Corpus Tools

\section{Projektkonzeption und -methode}

Das Projekt orientiert sich an den Methoden der historischen Wortbildungsforschung, wie sie in mehreren Korpusprojekten ursprünglich für das Deutsche entwickelt wurden und nun erstmals auf das Luxemburgische angewendet werden sollen. Die Methoden gehen auf die fünfbändige Deutsche Wortbildung der Innsbrucker Arbeitsstelle des Instituts für deutsche Sprache IDS zurück, die als synchron angelegte sprachstufenbezogene Analyse erstmals systematisch 
und korpusbezogen die morphologische und semantisch-funktionale Seite der Wortbildung der deutschen Gegenwartssprache beschrieb. Diese gesamthafte und systemorientierte Auswertung eines Textkorpus löste in der Wortbildungsforschung eine eher reihende, von den einzelnen Affixen ausgehende Darstellungsweise älterer historisch ausgerichteter Arbeiten wie Grimm (1826), Wilmanns (1896), Paul (1920), Kluge (1926), Henzen (1965) und Krahe/Meid (1969) ab. ${ }^{19}$ Die im Innsbrucker IDS-Projekt entwickelten Auswertungsmethoden und Darstellungsweisen wurden zunächst in verschiedenen Projekten zur Wortbildung des Frühneuhochdeutschen (cf. Müller 1993, Habermann 1994 und Thomas 2002) an die Erfordernisse älterer Sprachstufen angepasst und später auch auf das Mittelhochdeutsche ${ }^{20}$ übertragen.

Als Ziel dieser neueren historischen Wortbildungsforschung formulierte Solms (1998: 607) bezogen auf das Deutsche ,eine für alle Sprachepochen des Dt. gleichermaßen zu erarbeitende synchrone, korpusbezogene, funktional orientierte, das systemische Zusammenspiel der Wortbildungsmittel und -muster (als Konvergenz, Konkurrenz, Opposition) aufdeckende und die nachvollziehbaren Motivationsstrukturen zwischen den entstandenen Wortbildungskonstruktionen und ihren vorausliegenden Basiswörtern explizierende Wortbildung“‘. ${ }^{21}$

Den bisher vorliegenden Arbeiten zur Wortbildung des Mhd. bis Nhd. ist je nach Korpus und Fragestellung ein überschaubarer Zeitraum gemeinsam, der zwischen fünfzig (ca. 1250-1300 bei Ring 2008; Ganslmayer 2012) und etwa 300 Jahren (ca. 1050-1350 bei Klein/Solms/Wegera 2009) liegt, während der Sprachraum von einer Stadt (Nürnberg bei Müller 1993; Habermann 1994; Thomas 2002) bis zum gesamten hochdeutschen Sprachgebiet (Klein/Solms/Wegera 2009; Ring 2008; Ganslmayer 2012) reicht. Im vorzustellenden Projekt WBLUX ist der Untersuchungsraum im Vergleich zu den Mittelhochdeutsch-Projekten klein, der Zeitraum aber so groß, dass er in drei Zeitstufen unterteilt wird, die sich auch in der Überlieferungslage und in schreibsprachlichen Besonderheiten wiederfinden, cf. Tabelle 3:

${ }^{19}$ Zur Leistung dieser Arbeiten für die Erforschung der Wortbildung des Deutschen cf. ausführlicher Müller (1993: 15-16) und Solms (1998: 606-607).

${ }^{20}$ Cf. den Wortbildungsband der neuen mhd. Grammatik (Klein/Solms/Wegera 2009) sowie Ring (2008) und Ganslmayer (2012) zur Wortbildung in der Urkundensprache des 13. Jahrhunderts.

${ }^{21} \mathrm{Zu}$ den besonderen Herausforderungen einer historischen Wortbildungsforschung siehe auch Sieburg (2006). 


\begin{tabular}{|c|c|c|c|}
\hline Zeitstufe & I & II & III \\
\hline Jahrhunderte & $13 ., 14 ., 15$ & $16 ., 17 ., 18$. & $19 ., 20 ., 21$. \\
\hline $\begin{array}{l}\text { Untersuchungs- } \\
\text { raum }\end{array}$ & $\begin{array}{l}\text { Grafschaft bzw. Her- } \\
\text { zogtum Luxemburg }\end{array}$ & $\begin{array}{l}\text { Herzogtum Luxem- } \\
\text { burg }\end{array}$ & heutiges Luxemburg \\
\hline Korpus & Korpus 1 & Korpus 1 & Korpus 2 \\
\hline Domänen & $\begin{array}{l}\text { v. a. Recht u. Verwal- } \\
\text { tung }\end{array}$ & $\begin{array}{l}\text { v. a. Recht u. Verwal- } \\
\text { tung, Religion }\end{array}$ & $\begin{array}{l}\text { v. a. Literatur, Poli- } \\
\text { tik, Medien }\end{array}$ \\
\hline Schreibsprache & $\begin{array}{l}\text { moselfränkisch mit } \\
\text { hd. Einflüssen }\end{array}$ & $\begin{array}{l}\text { hochdeutsch mit } \\
\text { mslfrk. Resten }\end{array}$ & luxemburgisch \\
\hline Umfang (Tokens) & ca. $160^{\prime} 000$ & ca. $390^{\prime} 000$ & ca. $4^{\prime} 000^{\prime} 000$ \\
\hline
\end{tabular}

Tabelle 3: Zeitstufen 22

Ähnlich wie in Klein/Solms/Wegera (2009), die die mhd. Periode in Zeitschnitten von 50 bzw. 100 Jahren (nur Zeitraum I 1050-1150) weiter unterteilen, werden auch in den Korpora von WBLUX Zeitschnitte von 100 (Korpus 1), 50 (19. Jh.) bzw. 25 (20./21. Jh.) Jahren gesetzt, um Entwicklungen besser verfolgen zu können.

In der Darstellung der Ergebnisse hatte die Deutsche Wortbildung auf eine Dreiteilung gesetzt: ein „morphologischer Teil“" (z. B. Deutsche Wortbildung 3: 7) mit übergreifenden Kapiteln zur Phonologie, Affixvariation, Modifikation der Basen und zu Fugenelementen, ,morphembezogene Tabellen“" (z. B. Deutsche Wortbildung 3: 8), die die Ausdifferenzierung der Affixe nach Wortbildungsmustern tabellarisch darstellen, und ein nach Funktionen gegliederter ,Systematischer Teil“" (z. B. Deutsche Wortbildung 3: 8), der innerhalb der Funktionen nach den einzelnen Affixen untergliedert ist und den Schwerpunkt der Darstellung bildet. Auf der Grundlage dieser Aufteilung hat sich in den verschiedenen Projekten zur historischen Wortbildungsforschung in weitgehender Übereinstimmung eine zweiteilige Struktur mit einem semasiologischen Teil, der aus Artikeln zu den einzelnen Affixen mit Informationen zu Schreibvarianten, zur Basis- und Affixmorphologie sowie zur Wortbildungsfunktion besteht, und einem meist kürzeren onomasiologischen Teil, der aus funktionaler Perspektive u. a. Affixkonkurrenzen behandelt, herausgebildet. Die „morphembezogenen Tabellen“ aus der Deutschen Wortbildung finden sich in abgewandelter Form in den Affixkapiteln des semasiologischen Teils wieder. Dieser Darstellungsweise schließt sich auch das hier beschriebene Projekt weitgehend an mit Modifikationen, die v. a. wegen des langen Zeitraums notwendig wurden.

Ein Ziel des Projektes ist ein systematischer Überblick über die explizite Nominalderivation des Luxemburgischen und seiner moselfränkischen Vorgängervarietäten, der schließlich aus einer diachronen Blickrichtung zusammengeführt wird. Dabei stehen auch die Unterschiede zum und Wechselwirkungen mit dem Deutschen, der nächstverwandten Sprache, sowie mit dem Französischen im Blickpunkt. Neben diesen Aspekten der Mehrsprachigkeit und des Sprachkontakts werden Strategien zur Anpassung des Wortschatzes an veränderte Kommunikationsbedürfnisse, die sich durch die zunehmende Verwendung des Luxemburgischen in neuen Domänen ergeben, verfolgt.

\footnotetext{
${ }^{22} \mathrm{Zu}$ erwägen wäre für Zeitstufe I, vielleicht sogar für Zeitstufe II, wieweit hier der oben diskutierte Terminus „Altluxemburgisch“"vertretbar wäre.
} 


\section{Textbeispiele}

Du fëert mech och d'Meeschtesch

Mi dëw eran ann d'Donkelt,

Do huet eng naaß faul Wuurzel

An ängem Äck gefonkelt.

[...]

Ech sot: meng lëw Fra Echel,

$\mathrm{O}$ loßt mech hei erous;

Di Hetzt, dad feiregt Blenken:

Ech halen 't net më ous.

Michel Rodange: Renert oder De Fuuß an a Ma'nsgrëßt, 1872

(Hoffmann 1964: 526)

Der kurze Ausschnitt aus dem Versepos Renert von Michel Rodange, eine Bearbeitung von Goethes Reineke Fuchs (cf. Muller/Weber 2015), enthält drei Belege für nominale Ableitungen, die Feminina Meeschtesch ,Meisterin, Herrin', Hëtzt ,Hitze‘ sowie das Adjektiv feiereg ${ }^{23}$,feurig, feuerrot' (cf. LOD s. v. feiereg), die hier im Hinblick auf ihre Nähe zur deutschen Wortbildung betrachtet werden sollen. Feiereg/feireg ist mit dem Affix -eg vom Substantiv Feier ,Feuer' abgeleitet und entspricht damit formal dt. feurig zur Basis Feuer. Diese und andere Parallelen wie lux. -esch $\sim$ dt. -isch, lux. -lech $\sim$ dt. -lich, lux. on- $\sim \mathrm{dt}$. un-, lux. -ung $\sim$ dt. -ung, lux. -heet/-keet/-egkeet $\sim \mathrm{dt}$. -heit/-keit/-igkeit, lux. -er $\sim \mathrm{dt}$. -er sind wegen der engen Verwandtschaft und gemeinsamen Entwicklungsgeschichte des Luxemburgischen und Deutschen nicht verwunderlich. Feinere Unterschiede werden erst in der genauen Analyse sichtbar werden.

Studien wie der Wortbildungsband der neuen Mittelhochdeutschen Grammatik (Klein/Solms/Wegera 2012), deren Korpus das gesamte hochdeutsche Sprachgebiet erfasst, haben gezeigt, dass es in der Wortbildung in mhd. Zeit nur wenige regionale Unterschiede gibt. Die meisten Abweichungen vom restlichen Mhd. weist das Mittelfränkische auf, das im Grammatikkorpus v. a. durch ripuarische Texte, aber auch durch die aus Luxemburg stammende „Yolanda“ vertreten ist. Das zweite Beispiel aus dem „Renert“-Auszug Hëtzt ist eine alte Bildung, deren Suffix - $t$ sich auf ahd. -ida/-idi/-ido, mhd. -(e)de (cf. Klein/Solms/Wegera 2012: 72-76) zurückführen lässt. Es kommt zunächst im gesamten hochdeutschen Sprachraum vor und geht in einzelnen Lexemen wie Freude und Begierde in die nhd. Standardsprache ein, ist im Oberdeutschen aber in mhd. Zeit rückläufig. Im Mfrk. ist das Suffix schon in mhd. Zeit häufiger und es halten sich in den ripuarischen und moselfränkischen Dialekten (cf. Rheinisches Wörterbuch s. v. Hitze II: „Mosfrk hetst, -ø-“) sowie im Luxemburgischen viel mehr Bildungen, denen im Standarddeutschen Ableitungen auf -e entsprechen: z. B. lux. Hëtzt - dt. Hitze, lux. Längt - dt. Länge, lux. Déift - dt. Tiefe, lux. Héicht - dt. Höhe.

Wieder ein anderer Fall ist das dritte Beispiel Meeschtesch mit dem Movierungssuffix -esch, das mit dem Lautwandel /s/ > / $/$ nach /r/ auf älteres -se in Kombination mit auf $/ \mathrm{r} /$ auslautenden Basen zurückgeht (cf. Klein/Solms/Wegera 2012: 118-119): ${ }^{24}$ meister-se $\rightarrow$ lux. Meeschtesch. Dieses Suffix war wahrscheinlich von Anfang an regional auf das Westmitteldeutsche (und

\footnotetext{
${ }^{23}$ Das $-t$ in feiregt ist die Flexionsendung (Nom./Akk. Sg. neutr. des Adjektivs).

${ }^{24}$ Im Korpus der Mhd. Grammatik ist nur die Suffixvariante -sen, die vermutlich auf eine Verbindung mit dem südlich-hd. -in(ne) zurückgeht, belegt (cf. Klein/Solms/Wegera 2012: 118).
} 
Niederländische) begrenzt. Im Luxemburgischen steht es - teilweise in Kombination mit denselben Basen - in Konkurrenz zu -in, wie die beiden Beispiele von 1653 und 2008 zeigen:

[...] zu wißen die jenige welche keine zauberer oder zaubereinnen sein [...]

[...] wan Lannen Sun auff den dantzplatzen wehre mit einem grunen roch wehre gesehen worden und deßhalben voe eine zaubersche angesehen

[...] das ihr elteste dochter Magdallena [...] gesagt sie konnen auch zaubern, wisse auch die zauberßen dantzplatzen woll [...]

Hexenprozess Koerich 1653 gegen Lanen Sun (Archives de l'État à Arlon)

D’Familljeministerin Marie-Josée Jacobs huet Erklärungen ofginn iwwert d'Ursaachen vun de Problemer, mee awer och Hoffnung gemeet, dass et kéint an Zukunft besser goen. Iwwer 80.000 Telefonsappeller de Mount? D’Leit schaffe ganz gutt an der Kannergeldkeess, et ass awer nun mol quasi onméiglech, iwwer 80.000 Telefonsappellen de Mount unzehuelen an deene Leit ze hëllefen, esou d'Ministesch.

Nachrichtenportal rtl.lu, 23.10.2008

Weitere typisch luxemburgische Affixe, die wie -esch im Standarddeutschen entweder nicht oder nicht in derselben Funktion vorkommen wie im Luxemburgischen, sind -echt (Wourecht ,Wahrheit', Heemecht ,Heimat'), -ert (Fëmmert ,Raucher', Topert ,Trottel'), Ge-X-s (Gedrénks ,Getränke (Kollektivum)`, Gebrëlls ,Geschrei‘; cf. LOD s.vv.) und -elzeg (gréngelzeg ,grünlich‘, längelzeg ,länglich‘, cf. Gilles 2015).

Neben den deutsch-luxemburgischen Parallelen, die sich durch die enge Verwandtschaft erklären lassen, gibt es zahlreiche Fälle, die eher Folge des engen Sprachkontakts zwischen dem Luxemburgischen und Deutschen sind. Die Abgrenzung der beiden Phänomene ist schwierig, da sich die Ergebnisse des Sprachkontakts auf einem Kontinuum zwischen nicht integrierten Fremdwörtern und Lehnübersetzungen bewegen, die sich ausdrucksseitig nicht von nativen luxemburgischen Bildungen unterscheiden. Relativ klare Entlehnungen sind Fälle wie Moossnam ,Maßnahme', weil ein etymologisch entsprechendes Verb zu dt. nehmen im Luxemburgischen nicht existiert (cf. Mooss huelen ,Maß nehmen'). Lux. Beräich (,Bereich') lässt sich dagegen nur durch eine lauthistorische Analyse als Lehnwort erkennen. Da die meisten Luxemburgischsprecher mehrsprachig deutsch, französisch und luxemburgisch sind, stehen ihnen mehrere Varietäten zur Verfügung, die auch je nach Benennungsaufgabe ad hoc genutzt werden. Viele dieser Entlehnungen sind längst usuell geworden und ins Lexikon des Luxemburgischen eingegangen. Sofern sie morphologisch und semantisch motiviert sind, werden sie synchron als Teil der luxemburgischen Wortbildung analysiert. Entlehnungen aus dem Deutschen werden meist phonologisch und morphologisch integriert bzw. das deutsche Morphem durch das verwandte native Morphem substituiert.

An enger zweeter Etüd ronderëm d'Mobilfunktantenne sinn d'Auswierkunge gekuckt ginn, an enger Etüd vu Gomes et Peretta an der Stad Murcia a Spuenien. Do ass eng signifikant Bezéiung fonnt ginn, mat Middegkeet, Reizbarkeet, Kappwéi, Übelkeit, Appetitverloscht, Unbehagen a Bewegungsstörungen, an zwar och an deeneselwechte Beräicher ongeféier, déi ech virdru vun der Etüd vun der Uni Wien zitéiert hunn.

Parlamentsprotokoll, 13.05.2004

Der zitierte Abschnitt stammt aus einer Rede des Abgeordneten Jean Huss zum Thema möglicher Gefahren des Mobilfunkausbaus, wofür ein technisches und medizinisch-fachsprachliches 
Vokabular erforderlich ist. Da es sich um die Transkription einer mündlich vorgetragenen, aber wohl schriftlich vorbereiteten Rede handelt, ist weniger mit spontanen Transferenzen zu rechnen als in den Debattenteilen der Parlamentsprotokolle. Huss bezieht sich auf eine englischsprachige Studie und übersetzt die dort in einer Tabelle in dieser Reihenfolge gelisteten Symptome „Fatigue“, „Irritability“, „Headache“, „Nausea“, „Appetite loss““, „Discomfort“ und „Gait difficulty" (Navarro et al. 2003: 164) ins Luxemburgische, dabei ist Middegkeet mit der Suffixvariante -egkeet zur Basis midd ,müde' ganz nach luxemburgischen Wortbildungsregeln gebildet. Übelkeit, Unbehagen und Bewegungsstörungen erscheinen im Text als deutsche Adhoc-Übernahmen, obwohl Iwwelzegkeet und die Kompositionsglieder Beweegung und Stéierung auch in anderen Texten des Korpus belegt sind. Bei Reizbarkeet ist die adjektivische Basis reizbar belegt, der Diphthong lässt aber auf eine Entlehnung aus dem Deutschen schließen.

\section{$7 \quad$ Fazit}

Im Projekt „WBLUX“ steht zwar das Luxemburgische im Vordergrund, ohne Rückgriff auf das Deutsche und das (hier kaum behandelte) Französische lassen sich aber die Eigenheiten des luxemburgischen Wortbildungssystems nicht verstehen. Man kann davon ausgehen, dass das Luxemburgische im Laufe seiner Geschichte zur Modernisierung seines Wortschatzes die nächstverwandte und lange Zeit als Dachsprache fungierende deutsche Standardvarietät genutzt hat. Dieses Entlehnungsverhältnis bleibt auch mit der Entwicklung des Luxemburgischen vom Dialekt zur Ausbausprache bestehen.

\section{Literatur}

Deutsche Wortbildung. Typen und Tendenzen der Gegenwartssprache. Eine Bestandsaufnahme des Instituts für deutsche Sprache, Forschungsstelle Innsbruck. 5 Bände: 1. Hauptteil: Kühnhold, Ingeburg/Wellmann, Hans: Das Verb. Mit einer Einführung von Johannes Erben. Düsseldorf: Schwann 1973. 2. Hauptteil: Wellmann, Hans: Das Substantiv. Düsseldorf: Schwann 1975. 3. Hauptteil: Kühnhold, Ingeburg/Putzer, Oskar/Wellmann, Hans: Das Adjektiv. Düsseldorf: Schwann 1978. 4. Hauptteil: Ortner, Lorelies/Müller-Bollhagen, Elgin et al.: Substantivkomposita. Berlin/NewYork: de Gruyter 1991. 5. Hauptteil: Pümpel-Mader, Maria/Gassner-Koch, Elsbeth/Wellmann, Hans: Adjektivkomposita und Partizipialbildungen. Berlin/New York: de Gruyter 1992.

Dipper, Stefanie et al. (2013): „HiTS: ein Tagset für historische Sprachstufen des Deutschen“. JLCL 28/1: 85-137.

Ganslmayer, Christine (2012): Adjektivderivation in der Urkundensprache des 13. Jahrhunderts. Berlin/New York: de Gruyter. (= Studia Linguistica Germanica 97).

Gärtner, Kurt (2001): „Bruder Hermanns ,Leben der Gräfin Yolanda von Vianden“: Überlieferung und Edition“. In: Berg, Guy (ed.): Man mohte schrîven wal ein bi̊ch. Ergebnisse des Yolanda-Kolloquiums, 26.-27. November 1999, Luxemburg, Vianden und Ansemburg. Luxembourg, Institut Grand-Ducal, Section de Linguistique, d'Ethnologie et d'Onomastique: 39-51. (= Beiträge zur luxemburgischen Sprach- und Volkskunde 31, Sonderforschungsreihe Language and Culture in Medieval Luxembourg 3). 
Gilles, Peter (1998): „Die Emanzipation des Lëtzebuergeschen aus dem Gefüge der deutschen Mundarten“. Zeitschrift für deutsche Philologie 117: 20-35.

Gilles, Peter (1999): Dialektausgleich im Lëtzebuergeschen. Zur phonetisch-phonologischen Fokussierung einer Nationalsprache. Tübingen: Niemeyer. (= Phonai 44).

Gilles, Peter (2011): „Mündlichkeit und Schriftlichkeit in der luxemburgischen Sprachgemeinschaft“. In: Mein, Georg/Sieburg, Heinz (eds.): Medien des Wissens. Interdisziplinäre Aspekte von Medialität. Bielefeld, transcript: 43-64. (=Literalität und Liminalität 4).

Gilles, Peter (2015): "Evaluative morphology in Luxembourgish". In: Grandi, Nicola/Kortvelyessy, Livia (eds.): Edinburgh Handbook of Evaluative Morphology. Edinburgh, Edinburgh University Press: 262-268.

Grimm, Jacob (1826): Deutsche Grammatik. Zweiter Theil. Göttingen: Dieterich.

Habermann, Mechthild (1994): Verbale Wortbildung um 1500. Eine historisch-synchrone Untersuchung anhand von Texten Albrecht Dürers, Heinrich Deichslers und Veit Dietrichs. Berlin/New York: de Gruyter. (= Wortbildung des Nürnberger Frühneuhochdeutsch 2).

Handschriftencensus. Eine Bestandsaufnahme der handschriftlichen Überlieferung deutschsprachiger Texte des Mittelalters. http://www.handschriftencensus.de [24.02.2015].

Hardt, Mathias (1870): Luxemburger Weistümer als Nachlese zu Jacob Grimms Weisthümern, gesammelt und eingeleitet von Hardt, Regierungsarchivar in Luxemburg. Luxemburg: Bück.

Helminger, Nico (1990): Frascht. Roman. Echternach: Editions Phi.

Henzen, Walter (1965): Deutsche Wortbildung. 3. Auflage. Tübingen: Niemeyer.

Hoffmann, Fernand (1964): Geschichte der Luxemburger Mundartdichtung. Erster Band: Von den Anfängen bis zu Michel Rodange. Luxemburg: Bourg-Bourger.

Klein, Thomas (2000): „Rheinische und westfälische Sprachgeschichte bis 1300“. In: Macha, Jürgen/Neuß, Elmar/Peters, Robert (eds.): Rheinisch-westfälische Sprachgeschichte. Köln etc.: 3-48. (= Niederdeutsche Studien 46).

Klein, Thomas/Solms, Hans-Joachim/Wegera, Klaus-Peter (2009): Mittelhochdeutsche Grammatik. Band III: Wortbildung. Bearbeitet von Thomas Klein, Aletta Leipold, Eckhard Meineke, Simone Schultz-Balluff, Heinz Sieburg, Hans-Joachim Solms, Sandra Waldenberger, Klaus-Peter Wegera. Tübingen: Niemeyer.

Kloss, Heinz (1978): Die Entwicklung neuer germanischer Kultursprachen seit 1800. 2. erweiterte Auflage. Düsseldorf: Schwann.

Kluge, Friedrich (1926): Nominale Stammbildungslehre der altgermanischen Dialekte. 3. Auflage bearbeitet von Ludwig Sütterlin und Ernst Ochs. Halle: Niemeyer.

LOD = Lëtzebuerger Online Dictionnaire. www.lod.lu [25.02.2015].

Majerus, Nikolaus (1956): Die Luxemburger Gemeinden nach den Weistümern, Lehenerklärungen und Prozessen. Bd. 3. Luxemburg: Sankt-Paulus-Druckerei.

Krahe, Hans/Meid, Wolfgang (1969): Germanische Sprachwissenschaft. III Wortbildungslehre. 7. Auflage bearbeitet von Wolfgang Meid. Berlin/New York: de Gruyter. (= Sammlung Göschen 2234).

Meyer, Antoine (1829): E' Schrek ob de' Lezeburger Parnassus. Vum A. Meyer. Luxemburg: Lamort. 
Moulin, Claudine (2006): „Grammatisierung und Standardisierung des Luxemburgischen. Eine grammatikographisch-sprachhistorische Annäherung“. In: Moulin, Claudine/Nübling, Damaris (eds.): Perspektiven einer linguistischen Luxemburgistik. Studien zu Diachronie und Synchronie. Heidelberg, Winter: 305-339. (= Germanistische Bibliothek 25).

Moulin, Claudine (ed.) (2009): Bruder Hermann von Veldenz, Leben der Gräfin Yolanda von Vianden. Textgetreue Edition des Codex Mariendalensis (Bibliothèque Nationale, Luxembourg, Ms. 860). Luxembourg: Institut Grand-Ducal, Section de Linguistique, d'Ethnologie et d'Onomastique. (= Beiträge zur luxemburgischen Sprach- und Volkskunde XXXVI).

Müller, Josef et al. (eds.) (1928-1971): Rheinisches Wörterbuch. Bearbeitet und herausgegeben von Josef Müller, ab Bd. VII von Karl Meisen, Heinrich Dittmaier und Matthias Zender. 9 Bände. Bonn: Fritz Klopp, Berlin: Fritz Klopp, Berlin: Erika Klopp.

Müller, Peter (1993): Substantiv-Derivation in den Schriften Albrecht Dürers. Ein Beitrag zur Methodik historisch-synchroner Wortbildungsanalysen. Berlin/New York: de Gruyter. (= Wortbildung des Nürnberger Frühneuhochdeutsch 1).

Müller, Roger/Weber, Josiane: „Rodange, Michel“. In: www.autorenlexikon.lu [11.02.2015].

Müller, Stefan (2012): „Annotation unter Berücksichtigung syntaktischen Wandels“. In: Lefèvre, Michel (ed.): Syntaktischer Wandel in Gegenwart und Geschichte. Akten des Kolloquiums in Montpellier vom 9. bis 11. Juni 2011. Berlin, Weidler: 363-388. (= Berliner Sprachwissenschaftliche Studien 28).

Nachrichtenportal rtl.lu: http://www.rtl.lu [24.05.2016].

Navarro, Enrique et al. (2003): "The Microwave Syndrome: A Preliminary Study in Spain". Electromagnetic Biology and Medicine 22: 161-169.

Palmer, Nigel (1991): „Von der Paläographie zur Literaturwissenschaft. Anläßlich von Karin Schneider, Gotische Schriften in deutscher Sprache, Bd. I“. Beiträge zur Geschichte der deutschen Sprache und Literatur 113: 212-250.

Paul, Hermann (1920): Deutsche Grammatik. Bd. V. Wortbildungslehre. Halle: Niemeyer.

Paul, Mhd. Gr. = Paul, Hermann (1881/2007): Mittelhochdeutsche Grammatik. 25. Aufl., neu bearbeitet von Thomas Klein, Hans-Joachim Solms und Klaus-Peter Wegera. Mit einer Syntax von Ingeborg Schröbler, neu bearbeitet und erweitert von Heinz-Peter Prell. Tübingen: Niemeyer. (= Sammlung kurzer Grammatiken germanischer Dialekte A2).

Ravida, Fausto (2012): Graphematisch-phonologische Analyse der Luxemburger Rechnungsbücher (1388-1500). Ein Beitrag zur Historischen Stadtsprachenforschung. Heidelberg: Winter. (= Germanistische Bibliothek 43).

Ring, Uli (2008): Substantivderivation in der Urkundensprache des 13. Jahrhunderts. Eine historisch-synchrone Untersuchung anhand der ältesten deutschsprachigen Originalurkunden. Berlin/New York: de Gruyter. (= Studia Linguistica Germanica 96).

Russ, Charles V. J. (2000): "Word formation in Luxembourgish. Nominal and adjectival suffixes". In: Newton, Gerald (ed.): Essays on politics, language and society in Luxembourg. Lewiston, Edwin Mellen Press: 163-179.

Sánchez Prieto, Raúl (2008): „Die Wortbildung im Luxemburgischen“. Mikroglottika Yearbook 1: 157-169. 
Schanen, François/Zimmer, Jacqui (2005-2006): 1, 2, 3 Lëtzebuergesch Grammaire. Bd. 1: Le groupe verbal. Esch-sur-Alzette: Schortgen 2005. Bd. 2: Le groupe nominal. Esch-surAlzette: Schortgen 2006. Bd. 3: L'Orthographe. Esch-sur-Alzette: Schortgen 2006.

Schiltz, Pol/Kauthen, Pierre (eds.) (2009): Ephemeriden des Placidus Eringer. Übers. und Kommentar von Pol Schiltz und Pierre Kauthen. Luxembourg: Saint Paul.

Sieburg, Heinz (2006): „Zur Theorie und Praxis historischer Wortbildungsforschung“. In: Craemer, Susanne et al. (eds.): Europäische Begegnungen. Beiträge zur Literaturwissenschaft, Sprache und Philosophie. Festschrift für Joseph Kohnen. Luxemburg, Centre Universitaire de Luxembourg: 523-535.

Sieburg, Heinz/Weimann, Britta (2014): „Sprachliche Identifizierungen im luxemburgischdeutschen Grenzraum“. In: Wille, Christian et al. (eds.): Räume und Identitäten in Grenzregionen. Politiken - Medien - Subjekte. Bielefeld, transcript: 346-362.

Solms, Hans-Joachim (1998): „Historische Wortbildung“. In: Besch, Werner et al. (eds.): Sprachgeschichte. Ein Handbuch zur Geschichte der deutschen Sprache und ihrer Erforschung. 2. vollständig neu bearbeitete und erweiterte Auflage, 1. Teilband. Berlin/New York, de Gruyter: 596-610. (= Handbücher zur Sprach- und Kommunikationswissenschaft 2.1).

STATEC (ed.) (2014): Luxemburg in Zahlen. STATEC. Institut national de la statistique et des études économiques. Troisvierges. http://www.statistiques.public.lu/fr/publications/series/lux-chiffres-fr/index.html [25.02.2015].

Thomas, Barbara (2002): Adjektivderivation im Nürnberger Frühneuhochdeutsch um 1500. Eine historisch-synchrone Analyse anhand von Texten Albrecht Dürers, Veit Dietrichs und Heinrich Deichslers. Berlin/New York: de Gruyter. (= Wortbildung des Nürnberger Frühneuhochdeutsch 3).

Uhrmacher, Martin (2010): „Der Pyrenäenfrieden und seine Auswirkungen auf die Grenzziehung zwischen Frankreich und dem Herzogtum Luxemburg im Spiegel der Kartographie“. Hemecht 62/3: 463-492.

Weimann, Britta (2012): Moselfränkisch. Der Konsonantismus anhand der frühesten Urkunden. Wien etc.: Böhlau. (= Rheinisches Archiv 157).

Weimann, Britta (2013): „Überlegungen zur Entwicklung der Mündlichkeit und Schriftlichkeit in Luxemburg“. In: Sieburg, Heinz (ed.): Vielfalt der Sprachen - Varianz der Perspektiven. Zur Geschichte und Gegenwart der Luxemburger Mehrsprachigkeit. Bielefeld, transcript: 251-262. (= Interkulturalität. Studien zu Sprache, Literatur und Gesellschaf 3).

Wilmanns, Wilhelm (1896): Deutsche Grammatik. Gotisch, Alt-, Mittel- und Neuhochdeutsch. Zweite Abteilung: Wortbildung. Straßburg: Trübner. 